\title{
Affective Design Patterns in Computer Games. Scrollrunner Case Study
}

\author{
Grzegorz J. Nalepa \\ Jagiellonian University, \\ ul. Gołębia 24, 31-007 Kraków, Poland \\ AGH University of Science and Technology, \\ al. Mickiewicza 30, 30-059 Kraków, Poland \\ e-mail: gjn@agh.edu.pl
}

\author{
Barbara Giżycka \\ Jagiellonian University, \\ ul. Gołębia 24, 31-007 Kraków, Poland \\ e-mail: barbarag.cka@gmail.com
}

\author{
Krzysztof Kutt \\ AGH University of Science and Technology, \\ al. Mickiewicza 30, 30-059 Kraków, Poland \\ e-mail: kkutt@agh.edu.pl
}

\author{
Jan K. Argasiński \\ Jagiellonian University, \\ ul. Gołębia 24, 31-007 Kraków, Poland \\ e-mail: jan.argasinski@uj.edu.pl
}

\begin{abstract}
The emotional state of the user is a new dimension in human-computer interaction, that can be used to improve the user experience. This is the domain of affective computing. In our work we focus on the applications of affective techniques in the design of video games. We assume that a change in the affective condition of a player can be detected based on the monitoring of physiological signals following the James-Lange theory of emotions. We propose the use of game design patterns introduced by Björk and Holopainen to build games. We identify a set of patterns that can be considered affective. Then we demonstrate how these patterns can be used in a design of a scroll-runner game. We address the problem of the calibration of measurements in order to reflect responses of individual users. We also provide results of practical experiments to verify our approach.
\end{abstract}

\section{INTRODUCTION}

$\mathbf{I}$ $\mathrm{N}$ ORDER to improve usability and provide superior user experience we seek new sources of information that can be used in human-computer interaction. A new dimension of such an information is related to the emotional state of the user. The affective condition can be determined based on number of data, e.g. heart rate, expression of face, etc. This is the domain of the Affective Computing (AfC) paradigm, that was originally introduced 20 years ago [1]. We believe, that information regarding the affective condition of a user, can lead to a better understanding of interactions of users with machines, and interfaces.

In our work we focus on the applications of AfC techniques in the area of the design of video games. We assert that these techniques can improve the gaming experience of the player. In fact, the affective component can, and should be incorporated into the design process of video games. In order to do so, we propose the use of game design patterns as introduced in [2].

There are number of models of human emotions considered in psychology and adopted in AfC. In our work, we assume that a change in the affective condition of a player can be detected and identified based on the monitoring of physiological signals, following the James-Lange theory of emotions [3]. The new generation of miniaturized computer sensors can be used to measure such signals and deliver sensor data in real time to a gaming system. In fact, our priority is to focus on sensors that can be worn by a user, e.g. wearable wristbands. While such devices do not offer high quality of measurement, they are non intrusive and can be easily used by a player during a video game. Furthermore, they are not very expensive which makes them accessible to many players.

The original contributions of this paper are as follows: First we identify a set of game design patterns that can be considered affective, which means that they elicit an emotional response from the player. Then we demonstrate how these patterns can be used in a design of a simple yet illustrative game. Next, we address the problem of the proper calibration of measurements in order to reflect differences in responses of individual users. Finally, we provide results of practical experiments supporting our claims. Our work follows our early experiments with virtual reality discussed in [4].

The rest of the paper is composed as follows. In Section II we briefly introduce the affective computing paradigm, and selected methods and models we use in our work. In Section III we discuss the concept of patterns in game design. We introduce our motivation in Section IV. Then in Section V we demonstrate a practical design example of a scroll-runner game with affective components. To verify our assertions, we planned and conducted practical experiments described in Section VI. We briefly discuss other works related to our research in Section VII. The paper ends with summary and plans for future works in Section VIII.

\section{AfFective COMPUting}

Affective computing is a paradigm originally proposed in 1997 by Rosalind Picard from MIT Media Lab [1]. It uses results of biomedical engineering, psychology, and artificial intelligence. It aims at allowing computer systems to detect, 
use, and express emotions [5]. It is a constructive and practical approach oriented mainly at improving human-like decision support as well as human-computer interaction. AfC puts interest in design and description of systems that are able to collect, interpret, process (ultimately - simulate) emotional states (affects). Assuming that emotions are both physical and cognitive, they can be studied interdisciplinary by computer science, biomedical engineering and psychology. For affective computing there are two crucial elements to be considered: modes of data collection and ways of interpreting them in correlation with affective states corresponding to emotions.

The first is carried out by selection of methods for detecting information about emotions, using sensors capturing data about human physical states and behaviors. Today most often processed information is about: speech, body gestures and poses, facial expressions, physiological monitoring (blood pressure, blood volume pulse, galvanic skin response). In our research we focus on the last source of signals, and we assume a range of wearable physiological sensors.

The second crucial element of affective computing paradigm relies on application of selected algorithms on acquired data to develop models of interpretation for affective states. State of the art methodologies assume the use of the full range of available methods of data classification and interpretation [5].

Computational models of emotions derive from our interpretation of psychological states and cognitive processes and are in essence a way to describe relation between those two phenomena. Affective computing makes its own use of models by applying them to bio-physiological data obtained from sensors in such a way that they can be used in specific software. Defining "emotion" is a challenge. Modern theories of emotions have their origin in 19th century. William James theorized about affects in terms of reactions to stimuli. He was precursor to appraisal theory which is among most popular in the community of computational emotional modeling [6], [7], [8]. One of the most popular appraisal theories is OOC [9] which categorizes emotion on basis of appraisal of pleasure/displeasure (valence) and intensity (arousal). These are quantifiable values that can be measured and processed ascribing different kinds of emotions (i.e. positive self-attribution of intensive value might be interpret as "pride"). Another important set of theories of emotions is less about discrete states and more about relational affect states tracked on a number of continuous dimensions [10]. Dimensional models similarly to appraisal map emotion-evoking impulses and states triggered. Popular PAD theory [11] considers Pleasure, Arousal and Dominance dimensions. Different theories of emotion lead to various models which in turn lead to variety of emotion-oriented systems. Good example is EMA [10] - a system implemented on the Soar cognitive architecture which explains dynamic affects through a sequence of triggers. Another system is WASABI [12] believed to be one of most general models of emotion (mainly for simulation).

Nowadays most popular GUIs are based on windows, menus and forms. Natural User Interfaces (NUI) are meant to reduce the barriers for users even further while empowering them to perform more complicated tasks smoothly. In Adaptive User Interfaces (AUI) paradigm the assumption is that interfaces are subjects to modification as a result of interaction with the user. Adaptation can take place on many levels. Most often it means dynamically scalable quantity of displayed information depending on users demands and capabilities.

User interfaces based on emotion processing are in a way fusion of NUI and AUI. They are more "natural" than classic metaphor-based UIs because for humans "emotive" means natural. First, we have to keep in mind that in today's practice of UI design "emotive interface" means above all the use of techniques that cause emotions in receiver by using classical means of expression and communication. For us much more interesting will be the inclusion of affective processes at interfaces at the level of affective feedback for user.

In our work, we assume it is possible to identify a highlevel emotional state from low-level sensory data. We believe, that Jesse Prinz's Embodied Appraisal Theory [13] may be useful to reach this goal. According to it, emotions are build up by two parts: (a) form - bodily changes perception (as in the classical James-Lange theory [3]) and (b) content relationship between agent and environment. As an example, faster heart rate (form) and perception of a loud sudden noise (content) build up fear.

In the next section we will discuss the design patterns in video games. Extending them with affective information will lead to new design methods for computer games.

\section{GAMe Design PATterns}

One of the most important elements when it comes to game design are mechanics. They are basic building elements of the whole game structure. If we define the game as "the voluntary attempt to overcome unnecessary obstacles" [14], there are two main factors required to establish "gaming situation". These are the mental attitude of a player entering the play ("lusory attitude") and rules prohibiting the use of more efficient for less effectual means ("lusory means"). The role of the designer of the game is to create the frames (game design) that make this kind of activity (play) possible and pleasurable. It takes place mostly through creating constitutive rules (mechanics) which activated by the player (dynamics) result in his or her affects (user's experience). Another basis for the engagement are storytelling and interface design, leading to the interactivity of play that causes player's immersion (see: [15]), but these are less important from our point of view. In conclusion mechanics are main factor constructing gameplay resulting in player's affective experience.

We can express rules of play as the verbs describing what player can do inside designed game system: he can "jump" the avatar, "hit" the enemy, "collect" the coins, "solve" the puzzles. Usually, the player is aware of most of the rules. Mechanics are very similar "verbal" constructs, but widely they include invisible principles of the game system, those implemented deeper in the software, frequently not displayed to the user interface. For example when we play an arcade game we are aware of the rules that tell us to shoot the enemies, but we 
usually do not know about details of the algorithms governing enemy A.I. Both shooting and A.I.'s behaviors are mechanics.

Particular game mechanics are a repeating phenomena that strongly depend on the genre. An interesting fact is that the dynamics (groups of mechanics activated by player) tend to have emergent properties - it means that different gameplays cannot be directly derived from basic mechanics. This case seems to be the main source of diversity in the gaming domain.

Repetitive nature of the mechanics is also an origin of the idea of game design patterns. There are few formulations of the concept of modeling and aligning basic elements of lusory structures. One of the most interesting is that proposed by S. Björk and J. Holopainen [2]. It is an approach to creating a language for talking about gameplay. As the authors state: "Essential to the discussion of gameplay are the different aspects of gameplay that can exist. Understanding these aspects is important if one wants to go into details about a specific game one is playing [...] there is a lack of terminology associated with the elements of gameplay. We offer a solution to this [...]" [2]. In Björk's and Holopainen's framework besides the description of the structural elements of overall gaming situation, we find a semi-formal exposition of particular instances of gameplay.

For example components such as boundary (rules, modes, goals of play), temporal (actions, events, closures), holistic (game instance, session, play session), structural (interface, game elements, players, facilitator) are described in details.

Game design patterns rely, as authors state: "on general descriptions of particular areas of gameplay without using quantitative measures" [2]. That means characterizations are simple definitions with detailed descriptions of relationships to other patterns. There are three basic types of relations: instantiation (patterns tend to be present together with others, some naturally imply the presence of others), modulation (patterns change or are changed by the presence of others), conflict (patterns render the presence of others impossible).

Basic design pattern template has the following form: Name (arbitrary); Core definition; General Description; Clues for Using the Pattern; Consequences; Relations; References. In [2] authors identified and described in detail over 290 basic patterns. The Game Design Patterns framework is specially designed to facilitate the work of designers and analysts. They allow for easy diagnose of potential problems and identification of nodes where emergent properties can occur.

One of the goals of our proposal is to distinguish an arbitrary set of design patterns which, we believe, can cause affective reaction of the player. Our intention is also to test if embodied dynamics resulting from this emotion-invoking mechanics can be observed on a physiological level and included in the main game loop. In the case of success, such operation should allow for creating more complex feedback loops in order of suppressing or amplifying evoked emotions.

\section{Motivation}

In our work we aim at demonstrating, how methods of affective computing can improve design of video games. We base our work on the game design patterns [2]. Specifically, we identify certain patterns that in our opinion can be considered "affective". We expect an emotional response of a player of a video game that contains these patterns. We believe that a thoughtful application of these patterns can lead to more immersive games and improve the gaming experience. The motivation of our work is to provide a method for identification, personalization, and application of the affective design patterns in video games.

Our objective is to provide integration of a sensory framework with a gaming environment. The framework will use wearable physiological hardware sensors for detection of user affects. Ultimately we aim at extending the game design process by the use of affective patterns, and introduce an affective loop in the gameplay. Based on our experience with various hardware, we selected two most promising ones. Empatica E4 is an advanced sensory wristband based on the technologies previously developed in the Affective Computing division of MIT Media Lab. Blood volume pulse and galvanic skin response sensors, as well as infrared thermopile and accelerometer are on board [16]. Microsoft Band 2 was developed mainly for tracking fitness goals. Equipped with optical heart rate, skin temperature and galvanic skin response (GSR) sensors as well as accelerometer, available through well documented Software Development Kit.

To verify our hypothesis we provide a two phase experiment. We assume, that the emotional responses of a player can be detected by the analysis of his physiological responses, such as heart rate. We expect there are individual differences of the responses of different players. To address this challenge we provide an initial calibration phase. During it, the player is exposed to a series of pictures evoking emotional response. These pictures are selected from the NAPS [17] data base ${ }^{1}$.

The second phase of the experiment uses a simple video game. In this game a series of design patterns is identified. We consider two variants of the game. The first basic variant does not include the patterns that we consider affective. In the affective variant we augment the game with design patterns resulting in stronger emotional responses of the player.

In the next section we discuss a design of a video game to be used in our experiments, with the use of design patterns.

\section{Practical Design of an Affective Game}

In order to implement our ideas, we started with the design of a simple video game. In the game some basic design patterns selected from Björk's and Holopainen's collection can be observed. The selected design patterns, both affective and non-affective ones, that were implemented in the game design are described in part V-B.

\section{A. Bridge Scroll-runner}

For the purposes of the study, a simple video game was designed. As video games are already well known as a mul-

${ }^{1}$ NAPS stands for Nencki Affective Picture System (http://lobi.nencki.gov. $\mathrm{pl} / \mathrm{research} / 8$ ) and is a set of affective images. The dataset consists of standardized images, as well as normative ratings of valence and arousal. It is freely available for noncommercial use by request. 
timodal, interactive tool for providing entertainment [18], the game design was inspired by platformer games. In this subgenre of action games, the player's goal is to control the character in order to traverse through the game world and complete subsequent levels. The character usually moves by running and jumping on the platforms placed along the way, hence the name "platformer" game. The player is challenged by various obstacles that are placed in the game world in order to prevent him from completing the game, such as holes, gaps, enemies, etc. Some popular examples of platform games are Donkey Kong (1981) or Super Mario Bros (1985).

The reason we chose a platformer game and not some other genre is that platform games are relatively easy to design and to control all their parameters, which includes applying and subtracting design patterns. Some patterns, such as collectibles in case of platform games, are already an inherent feature of the genre [19]. What is more, the game sessions can be short, but nonetheless entertaining for the player.

The concept of the game that was designed for the purpose of this study refers to traditional English nursery rhyme, „London Bridge is Falling Down”. The player's avatar (the character that the player controls in the game), is an English gentleman that needs to rush past the bridge that is falling apart. To control the avatar, the player uses left and right arrow keys on keyboard to navigate (the character proceeds from left to right), and the spacebar to jump. Therefore, the goal of the game is to complete the level within the specific limited time, and completing all of the five designed levels results in completing the game. In the basic variant of the game, the player completes the level by collecting required number of points, which increases per level (1000 points for the first level, 2000 points for the second level, etc.). The player collects the points, thereby increasing his score, by picking up diamonds that are placed along the way through the bridge. Each diamond increases the player's score by 10 . Current score is showed in the top left corner of the screen.

To make the game more challenging, each subsequent level has its time limit narrowed (90 seconds the first level, 75 seconds for the second level, 60 seconds for the third, etc.). The remaining time is showed in the top center part of the screen. If the player fails to collect the objective of collecting the number of points required for the certain level, the game is over - the bridge falls down, and the player has to start the game from the first level. Besides the diamonds that are worth 10 points each, the player can pick up the little clocks that grant additional 5 seconds to the remaining time. To enable the new player to familiarize with the game mechanics, a tutorial level is provided at the beginning of each game session. The tutorial level serves as a teaching ground for the player to learn the controls and objectives of the game. The player is given 5 minutes, within which he explores the level and the game elements. The tutorial level is completed when the score reaches 1000 points. The tutorial level appears each time the game is started (after pressing Enter key), but it can be skipped by pressing $[\mathrm{P}]$ on the keyboard when it is entered.

In the affective variant of the game, the gameplay is en- hanced by several elements that instantiate some game design patterns, which will be specified in the following section. Firstly, the score and time indicators are replaced by narrative description (,bad”, „nice”, ,good”, and ,excellent”) and the remaining time is represented by a circular indicator. Secondly, once every few seconds (ranging from 5 to 15), an event will occur that will decrease the current score (a brick falling from the top of the screen) or the remaining time (a crow approaching the character from the right part of the screen).

The game was designed using Game Maker Studio version 1.4. software (YoYo Games). All of the game sprites and materials (background, music) were either created by us (some sprites, using GraphicsGale Free Edition) or acquired from free online resources (e.g. http://freepik.com). In Figure 1 an example of design session of the game is shown. Actual gameplay of the affective variant of the game is presented in Figure 2.

\section{B. Identification of Patterns}

In the basic variant of the game that is used in this study, a subset of game design patterns from those proposed by [2] was identified by us. These are:

1) Alarms: the notifications that appear as a red text in the top center part of the screen (below the remaining time indicator) that inform the player that half of the remaining time has passed.

2) Avatar: the character that is controlled by the player.

3) Collecting: the objectives of the game are reached by collecting the diamond that increase the player's score.

4) Deadly Traps: events that result in game over. In case of this game it is the falling off the platform.

5) Dexterity Based Actions, Evade and Maneuvering: these patterns refer to the mode of play that poses some challenge for the player's dexterity and eye-hand coordination (moving and jumping to collect the diamonds, evading falling bricks and incoming enemies).

6) Levels: spatial structures within the game world. The player traverses through the level in order to complete the objective of the game.

7) Movement: pattern refers to the action of moving within the game world.

8) Pick-Ups: items (clocks) that the player may optionally gather while playing, which grant him additional time to complete the level.

9) Rhythm-Based Actions: the collectibles in the game are placed in a manner (clusters) that resembles the rhythm of the tune played in the background.

10) Score: the numerical representation of the player's progress in the game.

11) Single-Player Games: this pattern refers to the general mode where there is only one player in a game instance.

12) Time Limits: some games, such as "London Bridge" that is used in this study, rely on the fact that the time within which the player can complete some action or reach a certain state in the game is somehow restricted. 


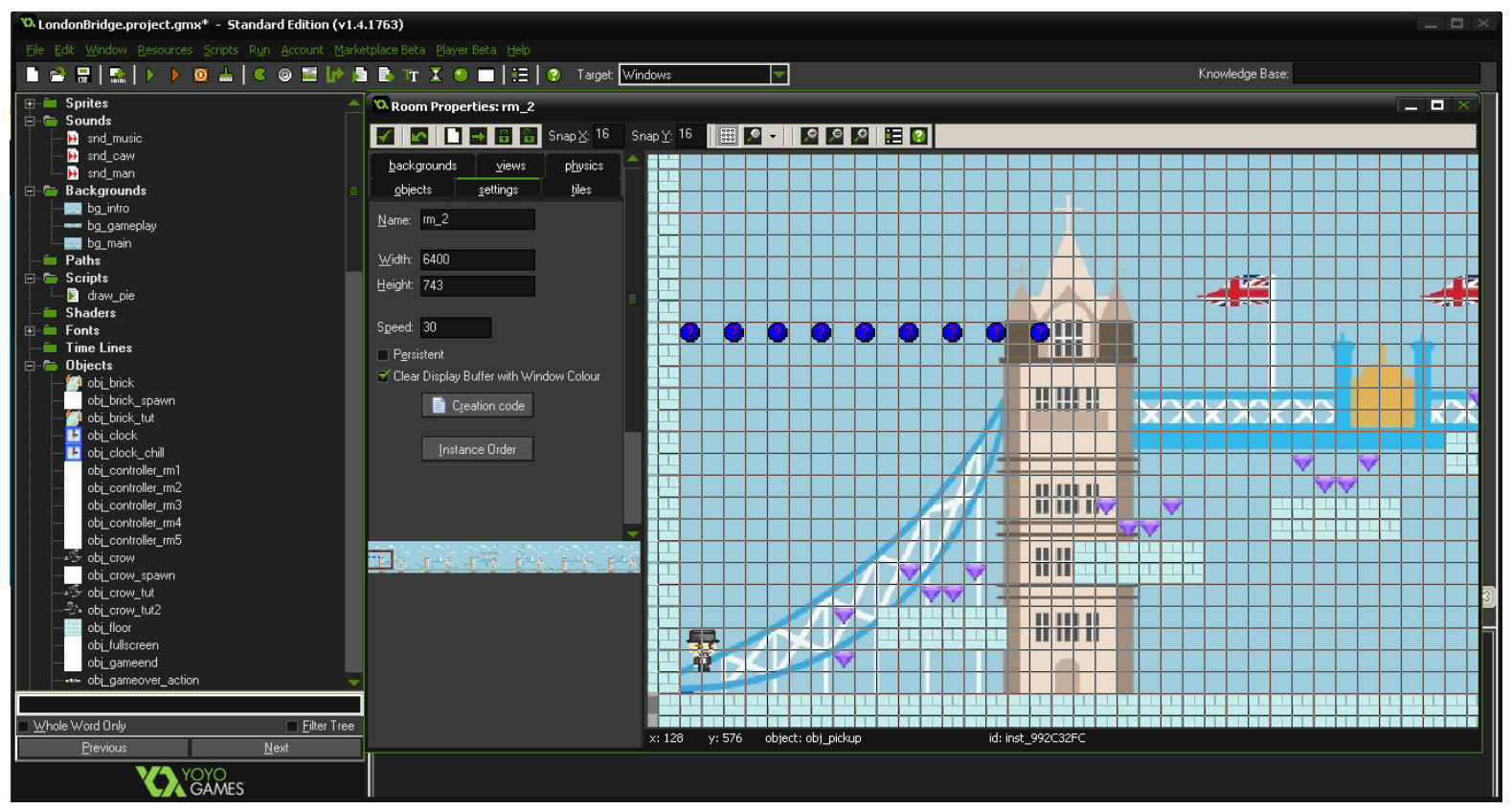

Fig. 1. Design of the scroll-runner game in the Game Makers Studio

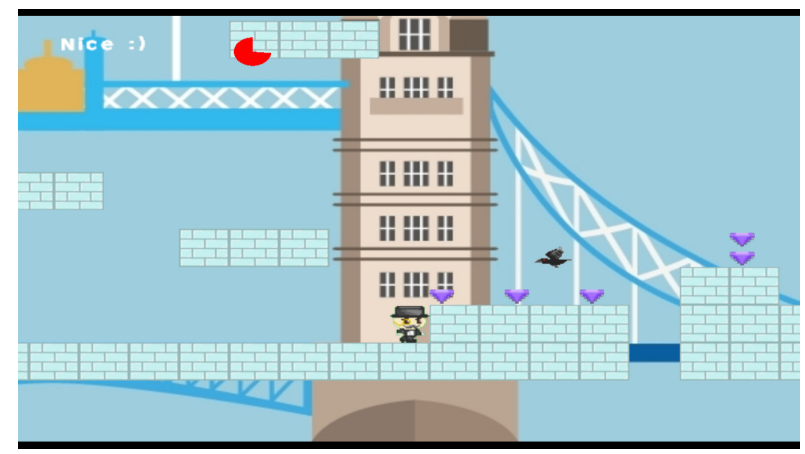

Fig. 2. Gameplay of the affective variant of the game. One can see the descriptive score (,nice”) and the crow approaching the character

\section{Affective Patterns}

In the affective variant of the game, some additional patterns are implemented:

1) Emotional Immersion: a higher level pattern that describes the player state that is hopefully reached when he becomes emotionally engaged in the gameplay.

2) Enemies and Obstacles: considered together, are elements that hinder the player trying to complete the game objective. Bricks and crows that consume score points and remaining time respectively.

3) Imperfect Information: occurs when some aspect of information about the game state is hidden from the player, for example - the exact score and time remaining.

In this study, player's affective responses - reflected by changes in his arousal, especially in heart rate and galvanic skin response - are anticipated in both variants of the game.
However, we hypothesize that the responses in the affective variant of the game will be stronger. We predict that exceptionally strong responses will appear when "Enemies and Obstacles" pattern occurs.

\section{EXPERIMENTS AND EVALUATION}

\section{A. Outline of the Calibration Phase}

Valence and arousal are two of several dimensions often used to characterize emotional experience (for overview of see [20]). Especially, valence differentiates states of pleasure and displeasure, and arousal contrasts states of low activation/relaxation and excitation [17], [21]. These dimensions are revealed in Autonomic Nervous System (ANS) activity, the part of nervous system responsible for unconscious autonomous functions like respiration and reflex actions. Research indicates that they can be measured by the use of ANS measures, inter alia, by the use of Heart Rate (HR) and Skin Conductance/Galvanic Skin Response (GSR) measures (for meta-analysis see [22]). Based on this research, the calibration phase was prepared. During it subjects are exposed to affective pictures from NAPS dataset. At the same time current levels of HR and GSR are collected by the wristband. Participants are also asked to evaluate the perceived arousal of each of the pictures. The goal of this phase is to combine physiological data with pictures' valence-arousal scores in order to prepare HR and GSR patterns as functions of them. These patterns will be used in the Gaming Phase as a reference to evaluate if affective design patters work as intended.

Experimental procedure was designed in the PsychoPyBuilder as shown in Figure 3. It was then detailed using the PsychoPy 2 (v 1.84.2) environment and executed on notebook with four cores processor $2.50 \mathrm{GHz}, 4 \mathrm{~GB}$ RAM working under 
the control of Windows 7 Professional $\mathrm{OS}^{2}$. Stimuli were presented on 15,6" notebook screen with 1366x768 resolution. Physiological data was collected by MS Band 2 and Empatica E4 bands. Bands were paired over Bluetooth with Samsung Galaxy J3 smartphone, on which custom application for data acquisition, developed by the authors, was installed. Data from smartphone and notebook is synchronized using the Lab Stream Layer ${ }^{3}$, a protocol for time-synced data transmission over local network, developed at the Swartz Center for Computational Neuroscience, University of San Diego.

Affective stimuli was a subset of Nencki Affective Picture System. Pictures in this set have assigned valence and arousal scores on 7 point scale [1-7] [17]. It was arbitrarily divided into three same-length intervals: low $[1,3)$, neutral $[3,5]$ and high $(5,7]$. Then, based on these intervals, group of pictures were selected in order to cover the groups of interest: (a) neutral valence and neutral arousal -10 pictures $(+6$ pictures for training session), (b) low valence and high arousal - 15 pictures, (c) high valence and low arousal - 15 pictures, (d) high valence and high arousal - 15 pictures. NAPS has few low valence and low arousal pictures, therefore this group was not represented in the selected subset.

The experiment is done individually and takes about 22 minutes. At the beginning, subject seats comfortably in front of the notebook. The wrist band is then placed on the less used hand, to minimize muscle artifacts associated with user interaction with procedure. PsychoPy procedure starts with instruction and training session with 6 neutral images. Each stimuli is presented for 3 seconds, then it disappears and subject has 5 seconds to evaluate the arousal on 7-levels scale $[1,7]$. After that another image appears without any pause. Training session ends with time for questions to experimenter. Then main session starts with 30 seconds of blank screen to get baseline HR and GSR recordings during inactivity. After that subjects is exposed to 3 series of 18 pictures. Series are separated by the 30 seconds breaks.

\section{B. Outline of the Gaming Phase}

After the subject completes the task in the calibration phase, the experimenter immediately runs the „London Bridge” game on the same machine for the subject. The subject still has the wristband on while the synchronization between the smartphone and the notebook is maintained. Before the Gaming Phase, the subject is randomly ascribed either to control group, where subjects play the basic game variant, or experimental group, where subjects play the affective game variant. After pressing Enter key on the notebook to start the game, a 30 seconds of blank screen is presented to get the baseline HR and GSR recordings for the beginning of the Gaming Phase. After 30 seconds, tutorial level appears and the subject is allowed to freely explore the game mechanics, and then to play the game for the desired time, but not exceeding 20 minutes.

\footnotetext{
${ }^{2}$ PsychoPy (http://psychopy.org) is a standard software framework in Python to support a wide range of neuroscience, psychology and psychophysics experiments.

${ }^{3}$ See: https://github.com/sccn/labstreaminglayer.
}

\section{Practical Experiments}

The procedure of the Calibration Phase was applied in the first experiment that was conducted in late April 2017 (Eurokreator lab, Krakow, Poland). The subjects (6 persons) were two male and 4 female participants of the workshops which were held in the lab. The full procedure, consisting of Calibration Phase and Gaming Phase, has been conducted as the second experiment in mid-June 2017. The subjects ( 9 persons) were Polish students from AGH-UST.

At the time of experiments the Lab Stream Layer synchronization method was not running yet. Therefore, the synchronization between the phone and the notebook was acquired by the subject simultaneously pressing spacebar on the notebook and „START RECORDING” button on the phone. It should be noted that due to technical issues a total of 52 pictures was used in the first experiment instead of 54.

\section{Evaluation of Results}

During the calibration phase, the following data is acquired:

- NAPS Valence and arousal scores - shared with the NAPS set.

- Baseline HR and GSR - the average value of 30 seconds recordings of HR and GSR at the beginning of main session.

- Gamers arousal score - the rating given by the player after seeing each of the stimuli.

- HR and GSR reactions - the HR and GSR levels recorded from the appearance of the stimulus to the appearance of the next stimulus.

The ultimate goal of this phase is to prepare HR and GSR patterns as a function of valence-arousal scores. In order to achieve this, the following analyses are being conducted:

- Gamers arousal scores are compared with NAPS arousal scores as a simple verification of NAPS data. If these scores will be significantly different, further analyses will be conducted for both sets of values.

- Average absolute HR and GSR reactions are calculated for different valence, arousal and valence*arousal values (as described in Section VI-A). The statistical significance of the differences between them is checked.

- The same analysis are conducted for relative values: instead of taking HR and GSR values, the difference between actual reactions and baseline are considered.

- It is also checked "if change appears" in the moment of affective stimuli appearance: it is possible that band sometimes record growth and sometimes falls and there are no significant differences between reactions' levels, but there is always a change that can be used within game context.

Preliminary analyses were carried out. HR and GSR responses to the presented stimuli are depicted on the plots presented in Figure 4. Heart Rate varies as a function of arousal: higher arousal values are indicated by steeper HR changes than in the neutral condition and lower arousal values are associated with flatter HR changes. This is consistent 


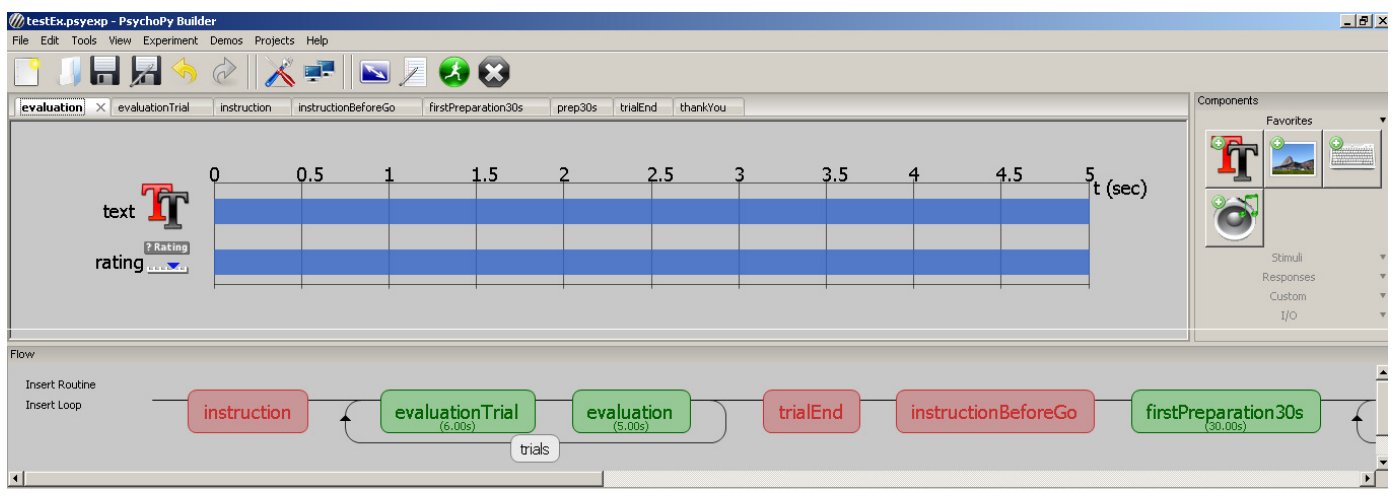

Fig. 3. PsychoPyBuilder Design of the experimental procedure for calibration
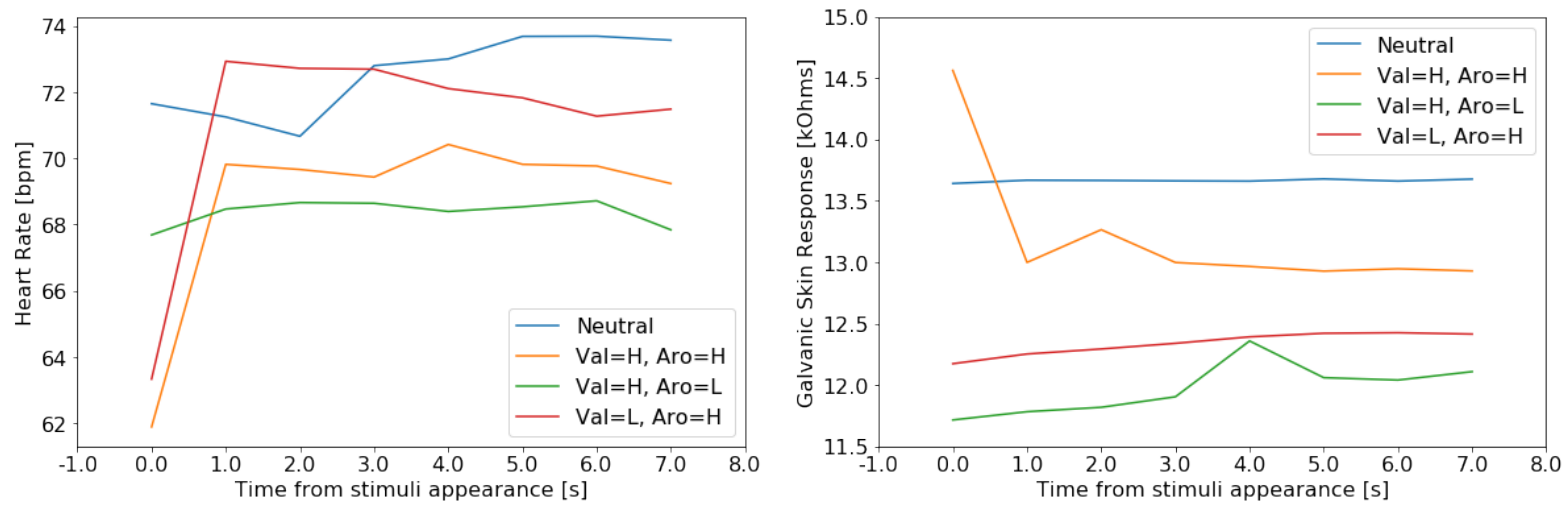

Fig. 4. HR and GSR responses with regard to the group to which the stimulus belonged $(\operatorname{Val}=$ Valence, $\mathrm{ArO}=\mathrm{Arousal}, \mathrm{H}=\mathrm{High}, \mathrm{L}=\mathrm{Low})$.

with both the general knowledge and the results of other research [20]: if a participant is more excited than his heart beats faster. On the other hand, Galvanic Skin Response varies as a function of valence. It shows changes to high valence values and no changes for others. These are important results because they point to the real utility of the budgetary wrist bands for differentiating emotional states.

As the data was collected just before the submission of the final version of this paper, a more exhaustive analyses leading to more accurate HR and GSR patterns identification are still being performed.

\section{RELATED RESEARCH}

In recent years there has been a lot of work related to the introduction of affective components into video games. Selected works are presented next.

In an early paper [23] an idea of enhancing biofeedback by placing it within a competitive virtual gaming environment was introduced. In fact, it was one of the first works to introduce affective feedback (loop). The virtual environment affects the players' level of relaxation while the levels of relaxation determine the outcome of the game. The most relaxed person wins the game. The study [24] investigates the hypothesis that the player's arousal will correspond with the pressure used to depress buttons on a gamepad. A practical video game was created to detect the force of each button press during play.
In [25] the authors examined phasic psychophysiological responses indexing emotional valence and arousal to different game events during the video game Monkey Bowling 2. Event-related changes in skin conductance, cardiac interbeat intervals, and other signals were recorded. Furthermore, game events elicited reliable valence- and arousal-related phasic physiological responses. The authors proposed to use the information on emotion-related physiological responses to game events or event patterns, to guide choices in game design.

The paper [26] describes an investigation into how realtime yet low-cost biometric information can be interpreted by computer games to enhance gameplay. The primary benefit of incorporating this technique into computer games is that game developers can offer control over direction of game play and game events to the player.

The paper [27] discusses how the emerging discipline of affective computing contributes to important elements of affective game design, with emphasis on the importance of modeling, including sensing and recognition of the players' emotions, generating 'affective behaviors'.

The paper [28] presents two studies that aim to realize an emotionally adaptive game. It investigates the relations between game mechanics, a player's emotional state and his/her emotion-data. In an experiment, one game mechanic (speed) was manipulated. Emotional state was self-reported in 
terms of valence, arousal and boredom-frustration-enjoyment. A number of (mainly physiology-based) emotion-data features were measured. Significant correlations were found between the valence/arousal reports and the emotion-data features.

As our work is not directly comparable in detail to any of these works, certain objectives are similar. What seems novel in our approach wrt to the state of the art is addressing specific affective game design patterns.

\section{FUTURE WORKS}

In this paper we discussed applications of affective computing techniques to the design of video games. We employed Jesse Prinz's Embodied Appraisal Theory that follows the assumptions of James-Lange theory of emotions. In this approach a change in the affective condition of a player can be detected and identified based of the monitoring of physiological signals of the player. In fact, we focuses on heart rate and galvanic skin response. We used game design patterns introduced by Björk and Holopainen.

Our contribution consists in the identification of a set of affective game design patterns. We demonstrated how these patterns can be used in a design of a scroll-runner game. We addressed the problem of differences in responses of individual users by the introduction of a proper calibration phase using NAPS pictures. Our approach is novel wrt to the state of the art, by addressing and using specific affective game design patterns.

Our objective was to provide integration of a sensory framework with a specific gaming environment to detect emotions. In this paper we discuss the use of the Game Maker environment. In the future we plan to support the Unity environment. We aim at extending the game design process by the practical use of affective patterns. In this way, the designer could introduce and control the affective loop in the game. Moreover, new series of experiments are planned. Finally, we are aiming to incorporate this work with a platform [29] combining affective computing with context-aware systems for ambient intelligence applications [30].

\section{ACKNOWLEDGMENT}

This work is supported by the Jagiellonian University and AGH University grants.

\section{REFERENCES}

[1] R. W. Picard, Affective Computing. MIT Press, 1997.

[2] S. Björk and J. Holopainen, Patterns in Game Design. Charles River Media, 2005

[3] W. James, "What is an emotion?" Mind, no. 34, pp. 188-205, 1884.

[4] G. J. Nalepa, J. K. Argasinski, K. Kutt, P. Wegrzyn, S. Bobek, and M. Z. Lepicki, "Affective computing experiments in virtual reality with wearable sensors. methodological considerations and preliminary results," in Proceedings of the Workshop on Affective Computing and Context Awareness in Ambient Intelligence (AfCAI 2016), ser. CEUR Workshop Proceedings, M. T. H. Ezquerro, G. J. Nalepa, and J. T. P. Mendez, Eds., vol. 1794, 2016.

[5] R. A. Calvo, S. K. D'Mello, J. Gratch, and A. Kappas, Eds., The Oxford Handbook of Affective Computing, ser. Oxford Library of Psychology. Oxford University Press, 2015.

[6] M. B. Arnold, Emotion and personality. Columbia University Press, 1960.
[7] R. S. Lazarus, Psychological stress and the coping process. McGrawHill, 1966.

[8] S. K. R., "Apprasial theory," in Handbook of cognition and emotion, D. T. and P. M., Eds. John Wiley and Sons, 1999, pp. 637-663.

[9] A. Orthony, G. Clore, and A. Collins, The cognitive structure of emotions. Cambridge University Press, 1988.

[10] S. C. Marsella and J. Gratch, "Ema: A process model of appraisal dynamics," Cognitive Systems Research, vol. 10, pp. 70-90, 2009.

[11] A. Mehrabian and J. A. Russell, An approach to environmental psychology. MIT Press., 1974.

[12] C. Becker-Asano and I. Wachsmuth, "Affective computing with primary and secondary emotions in a virtual human," Autonomous Agents and Multi-Agent Systems, vol. 20, pp. 32-49, 2009.

[13] J. Prinz, "Which emotions are basic?" in Emotion, evolution, and rationality, D. Evans and P. Cruse, Eds. Oxford University Press New York, 2004, pp. 69-88.

[14] B. Suits, The Grasshopper: Games, Life and Utopia. Broadview Press, 2005.

[15] B. Winn, "The design, play and experience framework," in Handbook of Research on Effective Electronic Gaming in Education. Hershey, 2009, pp. 1010-1024.

[16] R. W. Picard, "Recognizing stress, engagement, and positive emotion," in Proceedings of the 20th International Conference on Intelligent User Interfaces. ACM, 2015, pp. 3-4.

[17] A. Marchewka, Ł. Żurawski, K. Jednoróg, and A. Grabowska, "The Nencki Affective Picture System (NAPS): Introduction to a novel, standardized, wide-range, high-quality, realistic picture database," Behavior Research Methods, vol. 46, no. 2, pp. 596-610, 2014.

[18] S. Swink, Game Feel. A game designer's guide to virtual sensation. Elsevier, 2009.

[19] E. Andersen, Y.-E. Liu, R. Snider, R. Szeto, S. Cooper, and Z. Popović, "On the harmfulness of secondary game objectives," in Proceedings of the 6th International Conference on Foundations of Digital Games. ACM, 2011, pp. 30-37.

[20] I. B. Mauss and M. D. Robinson, "Measures of emotion: A review," Cognition and Emotion, vol. 23, no. 2, pp. 209-237, 2009.

[21] J. A. Russell, "A circumplex model of affect," Journal of Personality and Social Psychology, vol. 39, no. 6, pp. 1161-1178, 1980.

[22] J. T. Cacioppo, G. G. Berntson, J. T. Larsen, K. M. Poehlmann, and T. A. Ito, "The psychophysiology of emotion," in Handbook of emotions. Guildford Press, 2000, pp. 173-191.

[23] D. Bersak, G. McDarby, N. Augenblick, P. McDarby, D. McDonnell, B. McDonald, and R. Karkun, "Intelligent biofeedback using an immersive competitive environment," 2001, paper presented at the Designing Ubiquitous Computing Games Workshop at UbiComp 2001.

[24] J. Sykes and S. Brown, "Affective gaming: Measuring emotion through the gamepad,' in CHI '03 Extended Abstracts on Human Factors in Computing Systems, ser. CHI EA '03. New York, NY, USA: ACM, 2003, pp. 732-733.

[25] R. Niklas, S. Timo, L. Jani, K. Kari, and S. Mikko, "The psychophysiology of video gaming: Phasic emotional responses to game events," in DiGRA \&\#3905 - Proceedings of the 2005 DiGRA International Conference: Changing Views: Worlds in Play, 2005.

[26] K. M. Gilleade and A. Dix, "Using frustration in the design of adaptive videogames," in Proceedings of the 2004 ACM SIGCHI International Conference on Advances in Computer Entertainment Technology, ser. ACE '04. New York, NY, USA: ACM, 2004, pp. 228-232.

[27] E. Hudlicka, "Affective computing for game design," in Proceedings of the 4th International North American Conference on Intelligent Games and Simulation (GAMEON-NA), 2008.

[28] T. Tijs, D. Brokken, and W. Ijsselsteijn, "Creating an emotionally adaptive game," in Proceedings of the 7th International Conference on Entertainment Computing, ser. ICEC '08. Berlin, Heidelberg: SpringerVerlag, 2009, pp. 122-133.

[29] S. Bobek and G. J. Nalepa, "Uncertain context data management in dynamic mobile environments," Future Generation Computer Systems, vol. 66 , pp. $110-124,2017$.

[30] G. J. Nalepa, K. Kutt, S. Bobek, and M. Z. Lepicki, "AfCAI systems: Affective computing with context awareness for ambient intelligence. research proposal," in Proceedings of the Workshop on Affective Computing and Context Awareness in Ambient Intelligence (AfCAI 2016), ser. CEUR Workshop Proceedings, M. T. H. Ezquerro, G. J. Nalepa, and J. T. P. Mendez, Eds., vol. 1794, 2016. 